\title{
Perspectives from the Society for Pediatric Research: Decreased Effectiveness of the Live Attenuated Influenza Vaccine
}

\author{
Michelle A. Gill ${ }^{1}$ and Elizabeth P. Schlaudecker ${ }^{2}$
}

The intranasal live attenuated influenza vaccine (LAIV), FluMist, has been widely appreciated by pediatricians, parents, and children alike for its ease of administration. However, concerns regarding lack of effectiveness in recent influenza seasons led to the CDC Advisory Committee on Immunization Practices (ACIP) recommendation to administer inactivated influenza vaccines (IIVs), and not LAIV, during the 2016-17 and 2017-18 seasons. Given that data from previous years demonstrated equivalent and even improved efficacy of LAIV compared with $\| V$, these recent data were surprising, raising many questions about the potential mechanisms underlying this change. This review seeks to summarize the history of LAIV studies and ACIP recommendations with a focus on the recent decrease in vaccine effectiveness (VE) and discordant results among studies performed in different countries. Decreased VE for A/ H1N1pdm09 viruses represents the most consistent finding across studies, as VE has been low every season these viruses predominated since 2010-11. Potential explanations underlying diminished effectiveness include the hypothesis that prior vaccination, reduced thermostability of A/H1N1pdm09, addition of a fourth virus, or reduced replication fitness of $\mathrm{A} /$ H1N1pdm09 strains may have contributed to this phenomenon. Ongoing studies and potential alterations to LAIV formulations provide hope for a return of effective LAIV in future influenza seasons.

$T^{1}$ he potential for live attenuated influenza vaccines (LAIVs) to induce broader, longer-lasting protection than inactivated vaccines (1) combined with the ease of administration fueled intense interest in their development $(2,3)$. Reassortant influenza viruses prepared from cold-adapted $(c a)$ influenza $\mathrm{A}$ and $\mathrm{B}$ donor strains proved to be genetically stable (4), and commercial interest ensued. An early study conducted in 1985-1990 revealed that cold-adapted influenza vaccines against influenza $\mathrm{A}$ induced comparable or better immune responses to $\mathrm{A} / \mathrm{H} 1 \mathrm{~N} 1$ strains in seronegative children compared with inactivated vaccines, even after a single vaccine dose (5). Safety, immunogenicity, and efficacy studies conducted in the late 1990s provided promising results in both adults and children (6-9). In a much-cited large multicenter, double-blind, placebo-controlled trial that included over 1,500 children aged 15-71 months, vaccine efficacy of trivalent LAIV against culture-confirmed influenza was reported to be an encouraging $93 \%$ when vaccine and circulating virus strains were well matched (7). A subset of children (375 vaccinees and 169 placebo recipients) aged 6084 months completed a second year of this pediatric efficacy study. In season two, LAIV efficacy was $86 \%$ despite a mismatch between the influenza A (H3N2) vaccine component and circulating virus strains in that season. Overall, LAIV efficacy was $92 \%$ in preventing culture-confirmed influenza over the two-season study period (10). In addition, LAIV recipients experienced fewer episodes of otitis media (27\% reduction) and otitis media with concomitant antibiotic use (28\% reduction). Decreased fever and otitis media were also observed among vaccine recipients who experienced influenza infection.

LAIV was licensed by the Food and Drug Administration (FDA) in June of 2003. The Advisory Committee on Immunization Practices (ACIP) thereafter recommended LAIV as an option for vaccination of healthy persons aged 5-49 years (11). Considering the superior efficacy of LAIV over the inactivated influenza vaccine (IIV) demonstrated in several studies (12-14), the ACIP recommended preferential administration of LAIV to children aged 2-8 years when it was immediately available in 2014 (15), but this preferential recommendation was withdrawn in 2015 when concerns over decreased effectiveness of LAIV began to emerge (16). FDA approval for LAIV in adults was based on the results of two randomized, double-blind, placebo-controlled trials. The first study, designed to evaluate LAIV effectiveness in reducing influenza-like illness in a large number of healthy adults aged 18-49 years (17), demonstrated a $26 \%$ reduction in febrile upper respiratory illnesses in LAIV recipients compared with the placebo group. The second, smaller vaccine challenge study demonstrated efficacy of LAIV in healthy adults aged 18-41 years (85\% reduction in laboratory-documented influenza illness attributable to all three influenza strains combined) (8). Both of these trials were performed with LAIV

\footnotetext{
${ }^{1}$ Division of Infectious Diseases, Departments of Pediatrics, Internal Medicine, and Immunology, University of Texas Southwestern Medical Center, Dallas, Texas; ${ }^{2}$ Division of Infectious Diseases, Global Health Center, Department of Pediatrics, Cincinnati Children's Hospital Medical Center, Cincinnati, Ohio. Correspondence: Michelle A. Gill (Michelle. Gill@UTSouthwestern.edu)

Received 20 June 2017; accepted 15 September 2017; advance online publication 8 November 2017. doi:10.1038/pr.2017.239
} 
Table 1. Key definitions

\begin{tabular}{ll}
\hline Term & Definition \\
\hline Vaccine efficacy & $\begin{array}{l}\text { Percent reduction in disease incidence in a vaccinated group vs. unvaccinated group under optimal } \\
\text { conditions (such as RCT) }\end{array}$ \\
Vaccine effectiveness & $\begin{array}{l}\text { Percent reduction in the frequency of influenza-like illness among vaccinated vs. unvaccinated } \\
\text { individuals in "real-world" ambulatory settings } \\
\text { Test-negative case-control } \\
\text { design }\end{array}$ \\
& $\begin{array}{l}\text { Individuals meeting a pre-specified definition of illness (i.e., "case") undergo testing for influenza } \\
\text { Proportions of individuals with a history of vaccination among influenza test-positive and influenza } \\
\text { test-negative controls are compared }\end{array}$ \\
\hline
\end{tabular}

containing the seasonal H1N1 component, not the H1N1pdm09 component later associated with poor vaccine effectiveness.

\section{KEY DEFINITIONS: VACCINE EFFICACY VS. EFFECTIVENESS}

One factor complicating the interpretation and comparison of results across multiple studies of LAIV involves the different methodologies employed. Reported endpoints typically include measurements of either vaccine efficacy or vaccine effectiveness (Table 1), which represent very different outcomes. In simple terms, vaccine efficacy studies are designed to answer "Does the vaccine work?" within individuals. Efficacy studies utilize very stringent objective outcomes such as laboratory-confirmed diagnosis of influenza measured in highly controlled conditions such as randomized controlled trials (RCTs). In contrast, vaccine effectiveness studies address the more generalizable question "Does the vaccination work in real-world conditions?" by measuring the reduction of disease within a population in a more "realworld" setting $(18,19)$. Although each of these measures has associated advantages and disadvantages (reviewed in ref. 20), both are important in evaluating and monitoring vaccine performance.

Vaccine efficacy/effectiveness (abbreviated "VE" for both) is measured by calculating the risk of influenza disease among vaccinated and unvaccinated individuals and then determining the percentage reduction in risk of disease among vaccinated relative to unvaccinated individuals. $\mathrm{VE}$ is interpreted as the proportionate reduction in disease among the vaccinated group, with higher percentages conveying greater vaccine-associated disease protection (21).

\section{The Test-Negative, Case-Control Observational Study Design} Although vaccine efficacy is typically evaluated using the RCT study design, vaccine effectiveness is assessed with observational studies. The type of observational study design utilized to determine vaccine effectiveness is also critical in interpreting study outcomes. For the purposes of this review, which focuses on recent changes in LAIV effectiveness, most studies have employed the test-negative case-control design, considered as the gold standard for observational VE studies (2224). In the test-negative design (TND), individuals seeking medical care for acute respiratory illness are enrolled in ambulatory and hospital settings and tested for influenza virus infection with a highly sensitive and specific test. Those who test positive serve as influenza cases, and those who test negative are counted as controls. Vaccine coverage (the ratio of vaccinated to unvaccinated individuals) is then compared between those testing positive vs. those testing negative for influenza infection to estimate vaccine effectiveness (23). One advantage of the TND is that controls are drawn from the same source population as the influenza cases, facilitating rapid analyses of vaccine protection early in the influenza season (22). Additional advantages and disadvantages of the TND compared with other study designs are reviewed in detail in (25).

\section{REVIEW OF POST-LICENSURE EXPERIENCE: FROM EARLY SUCCESS TO RECENT DECREASED EFFECTIVENESS}

Following its licensure, LAIV was not initially approved for children $<5$ years of age because of an increased risk of asthma and reactive airway disease events observed in one study (26). However, after reviewing three pivotal vaccine trials, the FDA approved LAIV in 2007 for use among healthy children and adults from 2 through 49 years of age $(7,10,12,27)$. The pivotal Cold-Adapted Live Attenuated Influenza Vaccine, Trivalent (CAIV-T) Comparative Efficacy Study Group trial, and subsequent trials demonstrated superior efficacy of LAIV over IIV in children aged 671 months (12-14). This led the US ACIP to recommend preferential use of LAIV in 2014 for healthy children aged 2-8 years (15).

Since the 2004-05 influenza season, the Centers for Disease Control and Prevention (CDC) has conducted annual studies of influenza VE through the US Influenza Vaccine Effectiveness Network ("Flu VE Network"). Before the 2009 H1N1 pandemic, LAIV and IIV have been grouped together for VE estimates $(28,29)$. However, increasing use of LAIV among children and adolescents has allowed for individual estimates of VE for both LAIV and IIV during the 2009 H1N1 pandemic (30-32) and during subsequent seasons (33-35). These data demonstrated that both LAIV and IIV provided statistically significant and comparable protection against medically attended influenza illness in the outpatient setting during the 2010-11, 2011-12, and 2012-13 influenza seasons. VE point estimates against any influenza among children ranged from $45 \%$ (2012-13 season) to $71 \%$ (2010-11 season) for LAIV, compared with $48 \%$ (2012-13 season) to $71 \%$ (2010-11 season) for IIV $(33,35)$. A test-negative case-control study in Germany also demonstrated $84 \%$ VE for LAIV compared with $37 \%$ for IIV (36). These studies bolstered the 
Table 2. Influenza vaccine effectiveness by influenza season and vaccine type

\begin{tabular}{|c|c|c|c|c|c|}
\hline \multirow{2}{*}{$\begin{array}{l}\text { Influenza } \\
\text { Season }\end{array}$} & \multirow[t]{2}{*}{ Reference } & \multirow{2}{*}{$\begin{array}{l}\text { Number of } \\
\text { patients }\end{array}$} & \multirow[t]{2}{*}{ Influenza virus } & \multicolumn{2}{|c|}{ Adjusted overall VE (\%) ${ }^{\mathrm{a}}$} \\
\hline & & & & IIV & LAIV \\
\hline \multirow[t]{3}{*}{ 2009-10 } & Griffin et al. (30) & 6,757 & $\mathrm{~A} / \mathrm{H} 1 \mathrm{~N} 1 \mathrm{pdm} 09$ & $59 \%(26 \text { to } 77 \%)^{b}$ & $61 \%(12 \text { to } 82 \%)^{b}$ \\
\hline & Uzicanin et al. (31) & 1,621 & $\mathrm{~A} / \mathrm{H} 1 \mathrm{~N} 1 \mathrm{pdm} 09$ & $58 \%(-39$ to $87 \%)$ & $81 \%$ ( -37 to $97 \%)$ \\
\hline & Hadler et al. (32) & 807 & $\mathrm{~A} / \mathrm{H} 1 \mathrm{~N} 1 \mathrm{pdm} 09$ & $66 \%(<0$ to $99 \%)$ & $100 \%(<0$ to $100 \%)$ \\
\hline \multirow[t]{3}{*}{ 2010-11 } & Treanor et al. (33) & 4,757 & $\begin{array}{l}\text { Any influenza } \\
\text { virus }\end{array}$ & $62 \%$ (55 to $68 \%$ ) & $65 \%$ (46 to $77 \%$ ) \\
\hline & Chung et al. (50) & 2,177 & $\begin{array}{l}\text { Any influenza } \\
\text { virus }\end{array}$ & $66 \%$ (52 to $76 \%$ ) & $57 \%$ ( 25 to $76 \%$ ) \\
\hline & & 1,720 & $\mathrm{~A} / \mathrm{H} 1 \mathrm{~N} 1 \mathrm{pdm} 09$ & $75 \%$ (49 to $88 \%$ ) & $15 \%(-110$ to $65 \%)$ \\
\hline \multirow[t]{2}{*}{$2011-12$} & Ohmit et al. (34) & 4,771 & $\begin{array}{l}\text { Any influenza } \\
\text { virus }\end{array}$ & $40 \%(6 \text { to } 62 \%)^{c}$ & $61 \%(16 \text { to } 82 \%)^{c}$ \\
\hline & & & & $61 \%(28 \text { to } 79 \%)^{d}$ & $60 \%(-15 \text { to } 86 \%)^{d}$ \\
\hline \multirow[t]{5}{*}{$2012-13$} & McLean et al. (35) & 6,452 & $\mathrm{~A} / \mathrm{H} 3 \mathrm{~N} 2$ & $36 \%$ (15 to $51 \%)$ & $46 \%$ (13 to $66 \%)$ \\
\hline & & & $\mathrm{B} /$ Yamagata & $68 \%$ (54 to $77 \%$ ) & $53 \%$ (20 to $73 \%$ ) \\
\hline & Helmeke et al. (36) & 1,307 & $\mathrm{~A} / \mathrm{H} 1 \mathrm{~N} 1 \mathrm{pdm} 09$ & $-25 \%$ (-296 to $60 \%)$ & $39 \%(-176$ to $87 \%)$ \\
\hline & & & $\mathrm{A} / \mathrm{H} 3 \mathrm{~N} 2$ & $63 \%$ (-67 to $92 \%)$ & $84 \%(-27$ to $98 \%)$ \\
\hline & & & B & $39 \%$ (-66 to $78 \%)$ & Not available \\
\hline \multirow[t]{15}{*}{ 2013-14 } & Gaglani et al. (37) & 5,637 & $\mathrm{~A} / \mathrm{H} 1 \mathrm{~N} 1 \mathrm{pdm} 09$ & $60 \%$ (36 to $74 \%$ ) & $17 \%$ (-39 to $51 \%)$ \\
\hline & Caspard et al. (38) & 1,033 & $\mathrm{~A} / \mathrm{H} 1 \mathrm{~N} 1 \mathrm{pdm} 09$ & $74 \%$ (50 to $86 \%$ ) & $13 \%(-55$ to $51 \%)$ \\
\hline & & & $\mathrm{B} /$ Yamagata & $70 \%$ (18 to $89 \%)$ & $82 \%$ (12 to $96 \%)$ \\
\hline & Ohmit et al. (39) & 1,049 & A/H1N1pdm09 & $65 \%(-3 \text { to } 88 \%)^{c}$ & $82 \%(-65 \text { to } 98 \%)^{c}$ \\
\hline & & & & $78 \%(-150 \text { to } 98 \%)^{d}$ & $11 \%(-658 \text { to } 90 \%)^{d}$ \\
\hline & Skowronski et al. (40) & 1,700 & $\begin{array}{l}\text { Any influenza } \\
\text { virus }\end{array}$ & $76 \%(35 \text { to } 91 \%)^{e}$ & $83 \%(25 \text { to } 96 \%)^{e}$ \\
\hline & & & $\mathrm{A} / \mathrm{H} 1 \mathrm{~N} 1 \mathrm{pdm} 09$ & $75 \%(16 \text { to } 93 \%)^{e}$ & $86 \%(-11 \text { to } 98 \%)^{e}$ \\
\hline & Pebody et al. (43) & 4,442 & $A$ & $-69 \%(-409$ to $43.7 \%)$ & $31 \%(-29.5$ to $63.4 \%)$ \\
\hline & & & $\mathrm{A} / \mathrm{H} 3 \mathrm{~N} 2$ & $-73 \%$ (-457 to $46.2 \%)$ & $35 \%(-29.9$ to $67.5 \%)$ \\
\hline & & & $\mathrm{B} /$ Yamagata & $-123 \%(-1343$ to $65 \%)$ & $100 \%$ (17 to $100 \%)$ \\
\hline & Zimmerman et al. (41) & 9,311 & $\mathrm{~A} / \mathrm{H} 3 \mathrm{~N} 2$ & $15 \%$ (-16 to $38 \%)$ & $-3 \%(-50$ to $29 \%)$ \\
\hline & & & B/Yamagata & $40 \%$ (-20 to $70 \%)$ & $74 \%$ (25 to $91 \%)$ \\
\hline & McLean et al. (42) & 1,511 & $\begin{array}{l}\text { Any influenza } \\
\text { virus }\end{array}$ & $39 \%$ (18 to $54 \%)$ & $50 \%$ (27 to $66 \%)$ \\
\hline & & & $\mathrm{A} / \mathrm{H} 3 \mathrm{~N} 2$ & $40 \%$ (16 to $58 \%)$ & $30 \%$ (-6 to $54 \%)$ \\
\hline & & & $\mathrm{B} /$ Yamagata & $29 \%(-15$ to $56 \%)$ & $87 \%$ (63 to $96 \%$ ) \\
\hline \multirow[t]{7}{*}{$2015-16$} & Pebody et al. (44) & 176 & $\begin{array}{l}\text { Any influenza } \\
\text { virus }\end{array}$ & Not available & $54.5 \%$ (32 to $68 \%)$ \\
\hline & & & $\mathrm{A} / \mathrm{H} 1 \mathrm{~N} 1 \mathrm{pdm} 09$ & Not available & $48.3 \%$ (17 to $68 \%)$ \\
\hline & & & B & Not available & $70.6 \%$ (33 to $87 \%$ ) \\
\hline & Nohynek et al. (45) & 12,383 & $\begin{array}{l}\text { Any influenza } \\
\text { virus }\end{array}$ & $61 \%$ (31 to $78 \%)$ & $51 \%(28$ to $66 \%)$ \\
\hline & $\begin{array}{l}\text { ACIP presentation, Ambrose (46)(ICICLE Study } \\
\text { data) }\end{array}$ & 1,238 & $\begin{array}{l}\text { Any influenza } \\
\text { virus }\end{array}$ & $65 \%$ & $46 \%$ \\
\hline & & & $\mathrm{A} / \mathrm{H} 1 \mathrm{~N} 1 \mathrm{pdm} 09$ & $71 \%$ & $50 \%$ \\
\hline & & & B & $56 \%$ & $47 \%$ \\
\hline
\end{tabular}




\section{Review $\mid$ Gill and Schlaudecker}

Table 2 Continued

\begin{tabular}{|c|c|c|c|c|c|}
\hline \multirow{2}{*}{$\begin{array}{l}\text { Influenza } \\
\text { Season }\end{array}$} & \multirow[t]{2}{*}{ Reference } & \multirow{2}{*}{$\begin{array}{l}\text { Number of } \\
\text { patients }\end{array}$} & \multirow[t]{2}{*}{ Influenza virus } & \multicolumn{2}{|c|}{ Adjusted overall VE (\%) ${ }^{\mathrm{a}}$} \\
\hline & & & & IIV & LAIV \\
\hline & $\begin{array}{l}\text { ACIP presentation, Flannery and Chung(47) } \\
\text { (US Flu VE Network data) }\end{array}$ & 7,563 & $\mathrm{~A} / \mathrm{H} 1 \mathrm{~N} 1 \mathrm{pdm} 09$ & $63 \%$ & $3 \%$ \\
\hline & & & B & $65 \%$ & $-21 \%$ \\
\hline & & & $\mathrm{B} /$ Yamagata & $64 \%$ & $-4 \%$ \\
\hline & & & B/Victoria & $56 \%$ & $31 \%$ \\
\hline & $\begin{array}{l}\text { ACIP presentation, Flannery and Chung (47) } \\
\text { (Department of Defense Influenza } \\
\text { Surveillance Branch data) }{ }^{f}\end{array}$ & Not available & $\begin{array}{l}\text { Any influenza } \\
\text { virus }\end{array}$ & $66 \%$ & $53 \%$ \\
\hline & & & A/H1N1pdm09 & $68 \%$ & $15 \%$ \\
\hline & & & $\mathrm{H} 3 \mathrm{~N} 2$ & $82 \%$ & $47 \%$ \\
\hline & & & All B & $63 \%$ & $84 \%$ \\
\hline
\end{tabular}

ACIP, Advisory Committee on Immunization Practices; IIV, inactivated influenza vaccine; LAIV, live attenuated influenza vaccine; VE, vaccine effectiveness.

${ }^{a}$ Data in parentheses () represents $95 \%$ confidence intervals, and numbers in bold font represent significantly decreased VE for LAIV compared with IIV.

busing a 7-day rather than a 14-day interval before illness onset.

Vaccinees aged $2-8$ years.

dVaccinees aged $9-17$ years.

'Sample size was insufficient to support covariate adjustment, so data reported are crude VE estimates.

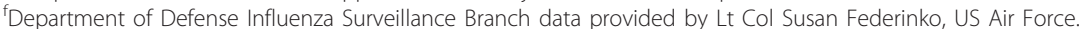

overall confidence in the effectiveness of LAIV compared with IIV.

Unfortunately, the data began to shift more significantly during the 2013-14 influenza season. The Flu VE Network indicated lower LAIV effectiveness among children in 201314 against illness due to $\mathrm{A} / \mathrm{H} 1 \mathrm{~N} 1 \mathrm{pdm} 09$ virus (37). This was demonstrated despite clear vaccine effectiveness of IIV (37). Analyzing the data by both vaccine type and influenza virus strain, VE against A/H1N1pdm09-related medically attended acute respiratory illness (MAARI) in LAIV recipients was $17 \%$, compared with $60 \%$ in IIV recipients (37). Caspard et al. (38) demonstrated similar results for A/H1N1pdm09, with $13 \%$ VE in LAIV recipients compared with $74 \%$ VE in IIV recipients. Of note, VE against $\mathrm{B} /$ Yamagata remained robust, with $82 \%$ VE in LAIV recipients and $70 \%$ VE in IIV recipients. Ohmit et al. (39) followed 232 households with 1,049 members, and LAIV was very effective against A/ H1N1pdm09 in children aged $2-8$ years ( $82 \% \mathrm{VE})$, but not in those aged 9-17 years (11\% VE), though numbers were small. In contrast, data from Canada demonstrated a crude VE for LAIV that was substantially higher than for IIV (83\% (95\% CI, -33 to $98 \%$ ) vs. $48 \%$ ( $95 \%$ CI, -16 to $90 \%)$ ), but confidence intervals were very broad secondary to the small sample size (40).

During the 2014-15 influenza season, VE of LAIV against B/Yamagata was sustained, with reported LAIV effectiveness of $74 \%$ (ref. 41 ), $87 \%$ (ref. 42), and $100 \%$ (ref. 43). In contrast, $\mathrm{VE}$ against $\mathrm{A} / \mathrm{H} 3 \mathrm{~N} 2$ was consistently low, with adjusted VE of $-3 \%$ (ref. 41 ), 30\% (ref. 42), and 35\% (ref. 43). However, a mismatch between the circulating $\mathrm{A} / \mathrm{H} 3 \mathrm{~N} 2$ virus and the $\mathrm{A} /$ H3N2 vaccine strain led to low VE for both LAIV and IIV during the 2014-15 season. In fact, adjusted VE estimates against influenza $\mathrm{A}, \mathrm{A} / \mathrm{H} 3 \mathrm{~N} 2$, and $\mathrm{B}$ were consistently higher for LAIV compared with IIV in the United Kingdom (43). In the study by McLean et al. (42), the overall VE against any influenza virus was higher for LAIV than IIV (50\% vs. 39\%). With the current data from the 2015-16 influenza season, studies from England (44), Finland (45), and the United States (46) have concluded that both vaccines continue to provide moderate VE against laboratory-confirmed influenza. However, further US data presented at the June 2016 ACIP meeting showed poor VE against A/H1N1pdm09 and H3N2, with conflicting VE against influenza B (47). In light of concerns regarding low effectiveness against A/H1N1pdm09 during the 2013-14 and 2015-16 seasons, ACIP made the interim recommendation that LAIV should not be used during the 2016-17 season (48), with subsequent recommendations to avoid LAIV during the 2017-18 season as well (49). These data are summarized in Table 2.

Further analyses of Flu VE Network data explored additional influenza-related outcomes, including influenzaassociated illness and community protection against influenza. In these comparisons of relative LAIV vs. IIV effectiveness, the odds of influenza-associated illness were significantly higher among LAIV recipients compared with IIV recipients aged 2-8 years during the 2013-14 influenza season only (OR 5.36; 95\% CI, 2.37-12.13) (50). This study also revealed that LAIV recipients had greater odds of illness due to influenza A/H1N1pdm09 in 2010-11 and 2013-14 (50). In a post-market observational study of 2013-14 LAIV in children aged 2-17 years, LAIV provided significant protection against $\mathrm{B} /$ Yamagata, but not A/H1N1pdm09 (38). During the 2014-15 season, a drifted influenza A/ H3N2 virus emerged that was associated with low VE in early influenza season estimates $(51,52)$. Among children aged 2-8 years, VE against $\mathrm{A} / \mathrm{H} 3 \mathrm{~N} 2$ was $15 \%$ for IIV and $-3 \%$ for 


\section{SPR perspectives on LAIV $\mid$ ReVIOW}

LAIV (41). Interestingly, VE against B/Yamagata was $40 \%$ for IIV and $74 \%$ for LAIV. In a cluster-randomized blinded trial conducted over three influenza seasons (2012-15) in 52 Huttrite colonies in Alberta and Saskatchewan, Canada, immunizing children with LAIV did not provide better community protection against influenza than IIV (53).

In summary, US data suggest that LAIV and IIV were both reasonably effective against influenza A/H1N1pdm09 during the 2009-10 season (30-32). The overall effectiveness of LAIV and IIV against all influenza viruses remained stable during the 2010-11 season (33), but LAIV effectiveness against A/ H1N1pdm09 was retrospectively found to be low (54). LAIV was again effective during the 2011-12 season (34), though no estimate was available for influenza $B$ because of low circulation of that virus (54). Both IIV and LAIV were ineffective against influenza A/H1N1pdm09 during the 201213 influenza season in Germany (36); VE was not calculated for the A/H1N1pdm09 strain from US data because of a low number of cases $(n=52)$ (35). VE data from 2013-14 season again demonstrated poor effectiveness against A/H1N1pdm09 in the United States $(37,38)$, but reasonable effectiveness against influenza B (38) in the youngest age group (2-8 years) (39) and in Canada (40). In 2014-15, LAIV was effective against B/Yamagata, but not A/H3N2 $(41,42)$. This pattern remained during the 2015-16 season, with low VE against A/ H1N1pdm09 and A/H3N2 strains, but overall effectiveness against B viruses $(44,45,47)$.

\section{CONUNDRUMS: DISPARITY AMONG LAIV EFFECTIVENESS STUDIES}

These conflicting data have introduced several significant questions and interpretations. The loss of LAIV effectiveness appears to be largely specific to the United States, with sustained VE in Germany, Canada, the United Kingdom, and Finland $(36,40,44,45)$. This suggests that certain vaccination or production strategies particular to the United States could contribute to decreased effectiveness. It has been hypothesized that overall high vaccination rates in the United States with subsequently high antibody titers at baseline may influence vaccine response. In addition, particular storage or handling methods in the United States may lead to inferior VE, as demonstrated in the post hoc analyses of a 2013-14 testnegative case-control effectiveness study (55).

It is notable that LAIV effectiveness diverges considerably by vaccine strain. In the second year of the 1996-1998 influenza vaccine trial, LAIV was $86 \%$ efficacious and more efficacious than IIV in preventing A/Sydney influenza, a variant not included in the influenza vaccine that year (10). During the 2009-10 pandemic H1N1 season, LAIV and IIV were both effective against A/H1N1pdm09 (30,31). However, LAIV effectiveness against A/H1N1pdm09 began to decline soon after the 2009-10 season. Because sample sizes were generally too small before the 2013-14 influenza season to detect VE differences by strain, the decline in LAIV's effectiveness against $\mathrm{A} / \mathrm{H} 1 \mathrm{~N} 1 \mathrm{pdm} 09$ was not noted until the 2013-14 season (37,38). Of note, the 2013-14 influenza season was the first time when influenza A/H1N1pdm09 viruses predominated in the United States since the 2009 H1N1 pandemic (56). In addition, quadrivalent LAIV (LAIV4) was introduced for the 2013-14 season, adding an additional influenza B virus to the previous trivalent LAIV. Compiled data from 2013-14, 2014-15, and 2015-16 seasons suggest that LAIV4 remained effective against influenza B strains (54), but questions about potential vaccine virus interference remain.

Table 3. Potential factors/mechanisms underlying decreased LAIV effectiveness

\begin{tabular}{|c|c|c|c|}
\hline Factor & & Potential mechanisms and available data & References \\
\hline Host-associated & Prior influenza vaccination & $\begin{array}{l}\text { Pre-existing influenza antibodies could interfere with replication of vaccine } \\
\text { virus strains. } \\
\text { Existing data do not support that this mechanism underlies the recent } \\
\text { decreased VE with LAIV, but suggest instead that prior immunization may } \\
\text { enhance LAIV performance. }\end{array}$ & $(42,52,58-61)$ \\
\hline \multirow[t]{2}{*}{$\begin{array}{l}\text { Vaccine virus- } \\
\text { associated }\end{array}$} & Reduced thermostability & $\begin{array}{l}\text { Heat exposure during LAIV distribution could result in degradation of } \\
\text { vaccine virus(es). } \\
\text { Reduced thermostability demonstrated for H1N1 pdm09 viruses in 2013/14 } \\
\text { and for A/California in laboratory experiments, leading to replacement with } \\
\text { A/Bolivia thermostable strain in 2015/16 LAIV }\end{array}$ & $(55,65)$ \\
\hline & Vaccine virus interference & $\begin{array}{l}\text { Potential that addition of second influenza B strain to LAIV could "interfere" } \\
\text { with immune response to other vaccine viruses. } \\
\text { No vaccine virus interference specific to the LAIV4 formulation has been } \\
\text { demonstrated and reduced VE against A(H1N1) pdm09 strains also } \\
\text { observed with LAIV3. }\end{array}$ & $(36,50,67,68,73)$ \\
\hline
\end{tabular}

LAIV, live attenuated influenza vaccine; VE, vaccine effectiveness. 


\section{POTENTIAL MECHANISMS UNDERLYING DECREASED VAC- CINE EFFECTIVENESS}

In considering potential factors underlying the recently observed reduction in LAIV VE, it is helpful to remember the general principle that live vaccines are thought to promote more intense innate responses compared with their inactivated vaccine counterparts. These brisk innate responses are stimulated by increased and prolonged viral antigen exposure, and thus require efficient replication of vaccine viruses within host cells (57). As such, any factor affecting the ability of vaccine viruses to bind, enter, and replicate within host cells could potentially affect the ensuing immune response and effectiveness of the vaccine. Several potential mechanisms contributing to decreased LAIV vaccine effectiveness have been proposed, and have been recently reviewed by Ambrose et al. (58). These are discussed below and divided into hostrelated and vaccine virus-associated factors (Table 3 ).

\section{Host-Associated Factor(s)}

Role of prior influenza vaccination. Although reduced vaccine effectiveness associated with prior influenza vaccination has been reported (52,59-61), this phenomenon has not been specifically linked to LAIV. The observations of recent reduced LAIV effectiveness in the United States, where vaccination rates have historically been high, with contrasting results in several European countries led to the hypothesis that pre-existing anti-influenza antibodies could potentially interfere with LAIV responses. This was evaluated in several studies in the 2013-14 and 2015-16 seasons, including the CDC Flu VE Network studies $(62,63)$ and the Influenza Clinical Investigation for Children (ICICLE) study (64); no deleterious effect of previous season influenza vaccination on LAIV VE was found. VE estimates actually trended higher among children who received influenza vaccination in the prior season in the ICICLE study, and this trend was also observed in another large pediatric study conducted in Finland (45). In addition, a recent study actually demonstrates improved LAIV performance against influenza A (H3N2) among patients who received prior vaccination in either of the two preceding seasons (42). Taken together, the available data do not suggest that prior influenza immunization/existing anti-influenza immunity represents a likely etiology of the recent decrease in LAIV effectiveness.

\section{Vaccine Virus-Associated Factors}

Reduced thermostability of the A/H1N1pdm09 vaccine virus. Data from the 2013-14 influenza season provided clinical evidence that the A/California (H1N1)pdm09 strain contained in LAIV was susceptible to heat degradation. In a comprehensive study conducted by MedImmune, associations between vaccine shipping conditions and LAIV lot effectiveness were examined within a test-negative case-control effectiveness study. Interestingly, a significant positive correlation was observed between the proportion of LAIV recipients testing positive for A/H1N1pdm09-associated illness and increased outdoor temperatures during time periods corresponding with truck unloading of LAIV lots by US distributors (55). Review of
LAIV VE study results revealed no significant effectiveness of LAIV against A/H1N1pdm09 for either the trivalent or the quadrivalent formulations of LAIV in the United States, respectively, in both the 2010-11 and 2013-14 influenza seasons, in contrast with significant effectiveness observed against $\mathrm{A} / \mathrm{H} 3 \mathrm{~N} 2$ and $\mathrm{B}$ strains during 2010-14. This link between poor VE of LAIV against A/H1N1pdm09 viruses with exposure of LAIV lots to temperatures above recommended storage conditions supported the concept of increased susceptibility of the A/California/7/2009 (H1N1pdm09) LAIV strain to thermal degradation. Laboratory studies, including experiments designed to simulate heat exposures occurring during US distribution confirmed reduced thermal stability of this strain (65), leading to the replacement of the A/ H1N1pdm09 LAIV strain with the more heat-stable A/ Bolivia strain in the 2015-16 formulation (38). Despite this change, reduced LAIV VE against A/H1N1pdm09 viruses was again observed in the $2015-16$ season $(48,66)$, suggesting that reduced thermostability does not explain recent observations of reduced LAIV VE against A/H1N1pdm09 strains (58).

Vaccine virus interference. Viral interference, defined as interference of replication by one virus strain when administered simultaneously with another virus strain to the same cell, is a phenomenon well documented among wild-type influenza viruses. The concept of vaccine virus interference is relevant to LAIV, as replication of vaccine viruses is required to elicit protective host immune responses (67). As evidence of decreased LAIV VE emerged, the possibility that the addition of a second influenza B strain to the LAIV4 formulation might interfere with immune responses to other vaccine viruses was proposed as a potential underlying cause of reduced VE. Studies were conducted in Senegal (68), Bangladesh (69), Germany (36), and the United States (50); however, all reported reduced VE against $\mathrm{A} / \mathrm{H} 1 \mathrm{~N} 1 \mathrm{pdm} 09$ strains even with trivalent LAIV formulations, although it is noted that the Senegal and Bangladesh studies were conducted with LAIV comprising Russian-derived vaccine viruses (based on A/Leningrad and B/ USSR master donor viruses). Prelicensure studies also demonstrated noninferiority of LAIV4 HAI antibody responses compared with trivalent LAIV responses among healthy children and adults (70-72). In addition, immunogenicity data from a MedImmune study presented to the FDA (study MI-CP185) compared four strain-specific serum HAI geometric mean titers post vaccination in quadrivalent vs. trivalent formulations of LAIV and found no evidence of viral interference attributable to the addition of a second influenza B strain (73). Vaccine virus interference specific to the quadrivalent formulation thus represents an unlikely etiology of the recently reduced VE observed with LAIV.

Investigations of potential viral interference between LAIV strains in studies conducted before the quadrivalent formulation of LAIV are also worth considering. In a viral interference-focused review of relevant pediatric and adult LAIV studies, Bandell and colleagues concluded that LAIV vaccine strains, given in either monovalent or trivalent 


\section{SPR perspectives on LAIV $\mid$ Review}

formulations, exhibited similar immunogenicity. Although viral shedding and/or immunogenicity of multivalent component strains was lower than that of the same strain administered in monovalent formulations in some of the studies reviewed, differences were not directly linked to viral interference and were hypothesized to be related to other factors including vaccine potency (67). Considering the evidence in its entirety, vaccine virus interference does not appear to be an intrinsic property of LAIV strains and is unlikely to be a primary cause of the current problem with poor LAIV VE.

Reduced replicative fitness. Factors contributing to the capacity of LAIV viruses to replicate, referred to as "replicative fitness", represent a focus of ongoing research. Emerging evidence from MedImmune laboratories suggests that poor replicative fitness of both the $\mathrm{A} / \mathrm{California}$ and more recent $\mathrm{A} /$ Bolivia (H1N1)pdm09 LAIV strains underlies recent observations of reduced vaccine effectiveness $(58,74)$. Reduced replication of $\mathrm{A} /$ California and A/Bolivia strains compared with pre-pandemic strains has been demonstrated in both human alveolar cell lines and primary human nasal epithelium air-liquid cultures. In addition, A/California and A/ Bolivia strains exhibit decreased binding to host cell influenza receptors and are less able to support multiple rounds of replication, important steps in eliciting the host immune response, compared with pre-pandemic strains (75). Although the mechanisms underlying this impaired replication fitness are unknown, they are postulated to be multifactorial and include such factors as hemagglutinin (HA) stability, HA activation $\mathrm{pH}$, and $\alpha 2-6$ sialic acid receptor binding. From the perspective of potentially overcoming this problem, preliminary data presented by MedImmune at the recent ACIP meeting in February 2017 are encouraging: a new H1N1 LAIV candidate strain, A/Slovenia, demonstrates increased replication in nasal epithelial cultures and no deficiency with multiple rounds of replication. It thus appears likely that this $\mathrm{A} / \mathrm{H} 1 \mathrm{~N} 1 \mathrm{pdm} 09$ strain will proceed to clinical trials planned for the upcoming influenza season. Although these preliminary data are encouraging, it is currently unclear whether the introduction of new $\mathrm{A} / \mathrm{H} 1 \mathrm{~N} 1 \mathrm{pdm} 09$ strains with improved replicative fitness will resolve the problem of decreased LAIV effectiveness. Additional studies, as well as funding to support these investigations, are required in order to make this determination.

Although the individual factors discussed above can all impact vaccine effectiveness, it is also possible that the etiology of decreased LAIV effectiveness is multifactorial, consisting of both known and currently unrecognized host and vaccine virus factors.

\section{SOCIETY FOR PEDIATRIC RESEARCH PERSPECTIVE: HOPE FOR THE FUTURE?}

Pediatricians, scientists, public health organizations, vaccine manufacturers, parents, and children alike share the frustration associated with the recent decrease in LAIV effectiveness and loss of this important tool in the prevention of influenza.
Despite these discouraging events, the prior history of success with LAIV paired with emerging new data from the manufacturer provides hope that the problems encountered in recent seasons can be overcome. Although discrepant results exist, decreased VE for A/H1N1pdm09 viruses represents the most consistent finding across studies, as the VE has been low every season in which these viruses predominated since 2010-11. Given that the predominant circulating influenza viruses change so frequently, identifying the problem with the specific $\mathrm{A} / \mathrm{H} 1 \mathrm{~N} 1 \mathrm{pdm} 09$ vaccine virus has been particularly difficult, and determining whether the strategies designed to overcome this are successful will be similarly challenging. That said, the recognition of poor replicative fitness of influenza $\mathrm{A} / \mathrm{H} 1 \mathrm{~N} 1 \mathrm{pdm} 09$ vaccine viruses and comprehensive investigations currently underway to overcome this have potential to improve LAIV effectiveness, although the timeframe for completion of such efforts is currently unclear.

The many advantages of LAIV highlight the importance of finding a solution to the current problems. LAIV has a history of superior efficacy, especially in children aged 6-71 months (12-14). Pediatric LAIV programs are both more costeffective and successful compared with IIV (76-78). In the setting of an influenza pandemic, LAIV offers shorter production time, great production yield (doses per egg), and more rapid, large-scale immunization than IIV (66). Despite its poor effectiveness against $\mathrm{A} / \mathrm{H} 1 \mathrm{~N} 1 \mathrm{pdm} 09$ viruses, there is a potential bystander cost to losing LAIV as an option during influenza $B$ and $A / H 3 N 2$ predominant seasons, given the superior VE of LAIV against these strains demonstrated in some studies (Table 2). LAIV also induces novel immune responses that may be superior to IIV responses, including Band T-cell responses that persist for at least 1 year in children (79). A recent study evaluating children vaccinated with LAIV at specific time points before elective tonsillectomies demonstrated that LAIV also enhances antibody and B-lymphocyte responses in palatine tonsils (80). The ability to boost durable, cross-reactive T-lymphocyte responses in children to genetically diverse influenza A strains to which the children were naive demonstrates the potential for LAIV to protect against newly emerging influenza strains (81). LAIV priming also induces greater inhibition of virus recovery upon LAIV challenge compared with IIV priming (82).

Point-by-point discussions of recent LAIV effectiveness data and the controversial issues surrounding application of these findings to public health decisions are published in the CDC ACIP meeting summary reports and make for interesting readings (83). Review of the ACIP deliberations reveals the many challenges associated with interpreting widely variable influenza VE obtained from different seasons, as well as the cautious and thoughtful approach taken by this committee in recommending that LAIV should not be used. One of the biggest hurdles in tracking VE of specific influenza vaccine viruses is the annual variation in the types of influenza viruses that circulate, with different viruses predominating nearly every influenza season. Recognition of 
the current problem with the influenza A/H1N1pdm09 virus component of LAIV was therefore delayed, since these viruses have only circulated at high levels in a few of the recent influenza seasons. As discussed at the 2017 ACIP meeting, VE data from the most recent 2016-17 influenza season are not expected to change the current recommendations, as influenza $\mathrm{A} / \mathrm{H} 3 \mathrm{~N} 2$ was the predominant virus in circulation in North America and Europe, whereas influenza A/H1N1 was a "non-player". Committee members noted that until there is an influenza season in which $\mathrm{A} / \mathrm{H} 1 \mathrm{~N} 1$ viruses play a significant role, there will be no way to evaluate whether planned changes to the A/H1N1 component of LAIV have resolved the problem. These discussions highlight the need to continue annual reassessment of LAIV effectiveness and the possibility that data needed to adequately evaluate the impact of planned changes in LAIV could take several influenza seasons to obtain.

Concerns about sustainability of the LAIV vaccine program from the standpoint of the manufacturer were also raised. The US Vaccines for Children Program reportedly ordered 14 million doses of LAIV for the 2016-17 influenza seasondoses that could not be used after the June 2016 ACIP recommendation $(66,84)$. The manufacturer of LAIV, MedImmune, a subsidiary of AstraZeneca, has thus far communicated an ongoing commitment to resolve the current problems with the $\mathrm{A} / \mathrm{H} 1 \mathrm{~N} 1 \mathrm{pdm} 09$ LAIV viruses, and to continue working with the CDC and ACIP in this effort. It is encouraging to note that FDA did not recommend any "specific regulatory action" after the 2016 ACIP decision, noting their perspective that "the benefits of FluMist Quadrivalent outweigh any potential risks" (85).

From the perspective of the Society for Pediatric Research, we remain hopeful that LAIV will be back. Although we endorse the latest ACIP recommendation, we also believe that the recent observations of poor vaccine effectiveness do not offset the many advantages of this vaccine. This stance is echoed by Penttinen and Friede's editorial (66), which highlights the critical need for comprehensive studies evaluating the viral, epidemiologic, and immune components of the decreased effectiveness of LAIV against A/H1N1 (pdm09) viruses in order to overcome this problem. We applaud the sustained efforts of public health organizations (CDC, ACIP, FDA, and World Health Organization) and the vaccine manufacturer in tackling these challenges. We encourage pediatricians, scientists, companies, and policy makers to continue working together in the ever-changing, ever-challenging race to prevent influenza infection, and hope that all will remain open-minded to the possibility of an improved and superior LAIV.

Lastly, motivated by our Society for Pediatric Research mission to "create a network of multidisciplinary researchers to improve child health", we are reminded of our responsibility to educate our patients and their families about the current situation and continue to recommend annual influenza immunization, even if our current vaccines and strategies are not as effective as desired. All is not lost, and alternative options (IIV) for the prevention of influenza infection remain, even in the midst of the current ongoing LAIV controversy. As stated by Dr David Kimberlin, representing the American Academy of Pediatrics at the June 2016 ACIP meeting: “The importance of preventing flu hasn't changed. What has changed is we have one less tool" $(83,86)$. As pediatricians and leaders in pediatric research, we remain focused on this message and hopeful that continued investigations will lead to the return of effective LAIV to our influenza prevention arsenals in the future.

Disclosure: The authors declare no conflict of interest.

\section{REFERENCES}

1. Clements ML, Murphy BR. Development and persistence of local and systemic antibody responses in adults given live attenuated or inactivated influenza A virus vaccine. J Clin Microbiol 1986;23:66-72.

2. Wareing MD, Tannock GA. Live attenuated vaccines against influenza; an historical review. Vaccine 2001;19:3320.

3. Murphy BR, Coelingh K. Principles underlying the development and use of live attenuated cold-adapted influenza A and B virus vaccines. Viral Immunol 2002;15:295-323.

4. Klimov AI, Egorov AY, Gushchina MI, et al. Genetic stability of coldadapted A/Leningrad/134/47/57 (H2N2) influenza virus: sequence analysis of live cold-adapted reassortant vaccine strains before and after replication in children. J Gen Virol 1995;76 (Pt 6): 1521-5.

5. Neuzil KM, Dupont WD, Wright PF, et al. Efficacy of inactivated and cold-adapted vaccines against influenza A infection, 1985 to 1990: the pediatric experience. Pediatr Infect Dis J 2001;20:733-40.

6. Mendelman PM, Cordova J, Cho I. Safety, efficacy and effectiveness of the influenza virus vaccine, trivalent, types $\mathrm{A}$ and $\mathrm{B}$, live, cold-adapted (CAIV-T) in healthy children and healthy adults. Vaccine 2001;19: 2221-6.

7. Belshe RB, Mendelman PM, Treanor J, et al. The efficacy of live attenuated, cold-adapted, trivalent, intranasal influenzavirus vaccine in children. N Engl J Med 1998;338:1405-2.

8. Treanor JJ, Kotloff K, Betts RF, et al. Evaluation of trivalent, live, coldadapted (CAIV-T) and inactivated (TIV) influenza vaccines in prevention of virus infection and illness following challenge of adults with wild-type influenza A (H1N1), A (H3N2), and B viruses. Vaccine 1999;18:899-906.

9. Piedra PA, Yan L, Kotloff K, et al. Safety of the trivalent, cold-adapted influenza vaccine in preschool-aged children. Pediatrics 2002;110:662-72.

10. Belshe RB, Gruber WC, Mendelman PM, et al. Efficacy of vaccination with live attenuated, cold-adapted, trivalent, intranasal influenza virus vaccine against a variant (A/Sydney) not contained in the vaccine. J Pediatr 2000;136:168-75.

11. Harper SA, Fukuda K, Cox NJ, et al. Using live, attenuated influenza vaccine for prevention and control of influenza: supplemental recommendations of the Advisory Committee on Immunization Practices (ACIP). MMWR Recomm Rep 2003;52 (RR-13): 1-8.

12. Belshe RB, Edwards KM, Vesikari $T$, et al. Live attenuated versus inactivated influenza vaccine in infants and young children. N Engl J Med 2007;356:685-96.

13. Fleming DM, Crovari P, Wahn U, et al. Comparison of the efficacy and safety of live attenuated cold-adapted influenza vaccine, trivalent, with trivalent inactivated influenza virus vaccine in children and adolescents with asthma. Pediatr Infect Dis J 2006;25:860-9.

14. Ashkenazi S, Vertruyen A, Aristegui J, et al. Superior relative efficacy of live attenuated influenza vaccine compared with inactivated influenza vaccine in young children with recurrent respiratory tract infections. Pediatr Infect Dis J 2006;25:870-9.

15. Grohskopf LA, Olsen SJ, Sokolow LZ, et al. Prevention and control of seasonal influenza with vaccines: recommendations of the Advisory 


\section{SPR perspectives on LAIV Review}

Committee on Immunization Practices (ACIP) - United States, 2014-15 influenza season. MMWR Morb Mortal Wkly Rep 2014;63:691-7.

16. Grohskopf LA, Sokolow LZ, Olsen SJ, et al. Prevention and control of influenza with vaccines: recommendations of the Advisory Committee on Immunization Practices, United States, 2015-16 influenza season. MMWR Morb Mortal Wkly Rep 2015;64:818-25.

17. Nichol KL, Mendelman PM, Mallon KP, et al. Effectiveness of live, attenuated intranasal influenza virus vaccine in healthy, working adults: a randomized controlled trial. JAMA 1999;282:137-44.

18. Fedson DS. Measuring protection: efficacy versus effectiveness. Dev Biol Stand 1998;95:195-201.

19. Weinberg GA, Szilagyi PG. Vaccine epidemiology: efficacy, effectiveness, and the translational research roadmap. J Infect Dis 2010;201:1607-0.

20. CDC Flu Vaccine Effectiveness: Questions and Answers for Health Professionals, 2017 (cited 22 May 2017). (https://www.cdc.gov/flu/ professionals/vaccination/effectivenessqa.htm).

21. CDC Principles of Epidemiology in Public Health Practice, Third Edition. An Introduction to Applied Epidemiology and Biostatistics. Lesson 3, Section 6, 2012 https://www.cdc.gov/ophss/csels/dsepd/ss1978/lesson3/ section6.html.

22. De Serres G, Skowronski DM, Wu XW, et al. The test-negative design: validity, accuracy and precision of vaccine efficacy estimates compared to the gold standard of randomised placebo-controlled clinical trials. Euro Surveill 2013;18:pii: 20585.

23. Sullivan SG, Feng S, Cowling BJ. Potential of the test-negative design for measuring influenza vaccine effectiveness: a systematic review. Expert Rev Vaccines 2014;13:1571-91.

24. Sullivan SG, Tchetgen Tchetgen EJ, Cowling BJ. Theoretical basis of the test-negative study design for assessment of influenza vaccine effectiveness. Am J Epidemiol 2016;184:345-53.

25. Treanor JJ. CLINICAL PRACTICE. Influenza Vaccination. N Engl J Med 2016;375:1261-8.

26. Bergen R, Black S, Shinefield H, et al. Safety of cold-adapted live attenuated influenza vaccine in a large cohort of children and adolescents. Pediatr Infect Dis J 2004;23:138-44.

27. Tam JS, Capeding MR, Lum LC, et al. Efficacy and safety of a live attenuated, cold-adapted influenza vaccine, trivalent against cultureconfirmed influenza in young children in Asia. Pediatr Infect Dis J 2007;26:619-28.

28. Belongia EA, Kieke BA, Donahue JG, et al. Effectiveness of inactivated influenza vaccines varied substantially with antigenic match from the 2004-2005 season to the 2006-2007 season. J Infect Dis 2009;199:159-67.

29. Belongia EA, Kieke BA, Donahue JG, et al. Influenza vaccine effectiveness in Wisconsin during the 2007-08 season: comparison of interim and final results. Vaccine 2011;29:6558-3.

30. Griffin MR, Monto AS, Belongia EA, et al. Effectiveness of nonadjuvanted pandemic influenza A vaccines for preventing pandemic influenza acute respiratory illness visits in 4 U.S. communities. PLoS ONE 2011;6:e23085.

31. Uzicanin A, Thompson M, Smith P, et al. Effectiveness of 1 dose of influenza A (H1N1) 2009 monovalent vaccines in preventing reverse-transcription polymerase chain reaction-confirmed H1N1 infection among school-aged children in maine. J Infect Dis 2012;206:1059-68.

32. Hadler JL, Baker TN, Papadouka V, et al. Effectiveness of 1 dose of 2009 influenza A (H1N1) vaccine at preventing hospitalization with pandemic H1N1 influenza in children aged 7 months-9 years. J Infect Dis 2012;206: $49-55$.

33. Treanor JJ, Talbot HK, Ohmit SE, et al. Effectiveness of seasonal influenza vaccines in the United States during a season with circulation of all three vaccine strains. Clin Infect Dis 2012;55:951-9.

34. Ohmit SE, Thompson MG, Petrie JG, et al. Influenza vaccine effectiveness in the 2011-2012 season: protection against each circulating virus and the effect of prior vaccination on estimates. Clin Infect Dis 2014;58:319-27.

35. McLean HQ, Thompson MG, Sundaram ME, et al. Influenza vaccine effectiveness in the United States during 2012-2013: variable protection by age and virus type. J Infect Dis 2015;211:1529-40.
36. Helmeke C, Grafe L, Irmscher HM, et al. Effectiveness of the 2012/13 trivalent live and inactivated influenza vaccines in children and adolescents in Saxony-Anhalt, Germany: a test-negative case-control study. PLoS ONE 2015;10:e0122910.

37. Gaglani M, Pruszynski J, Murthy K, et al. Influenza vaccine effectiveness against 2009 pandemic influenza $A(H 1 N 1)$ virus differed by vaccine type during 2013-2014 in the United States. J Infect Dis 2016;213:1546-56.

38. Caspard H, Gaglani M, Clipper L, et al. Effectiveness of live attenuated influenza vaccine and inactivated influenza vaccine in children 2-17 years of age in 2013-2014 in the United States. Vaccine 2016;34:77-82.

39. Ohmit SE, Petrie JG, Malosh RE, et al. Substantial influenza vaccine effectiveness in households with children during the 2013-2014 influenza season, when 2009 pandemic influenza A(H1N1) virus predominated. J Infect Dis 2016;213:1229-36.

40. Skowronski DM, Chambers C, Sabaiduc S, et al. Integrated sentinel surveillance linking genetic, antigenic, and epidemiologic monitoring of influenza vaccine-virus relatedness and effectiveness during the 20132014 influenza season. J Infect Dis 2015;212:726-39.

41. Zimmerman RK, Nowalk MP, Chung J, et al. 2014-2015 Influenza vaccine effectiveness in the United States by vaccine type. Clin Infect Dis 2016;63:1564-73.

42. McLean HQ, Caspard H, Griffin MR, et al. Effectiveness of live attenuated influenza vaccine and inactivated influenza vaccine in children during the 2014-2015 season. Vaccine 2017;35:2685-93.

43. Pebody R, Warburton F, Andrews N, et al. Effectiveness of seasonal influenza vaccine in preventing laboratory-confirmed influenza in primary care in the United Kingdom: 2014/15 end of season results. Euro Surveill 2015;20:30013.

44. Pebody R, Sile B, Warburton F, et al. Live attenuated influenza vaccine effectiveness against hospitalisation due to laboratory-confirmed influenza in children two to six years of age in England in the 2015/16 season. Euro Surveill 2017;22:30450.

45. Nohynek H, Baum U, Syrjanen R, et al. Effectiveness of the live attenuated and the inactivated influenza vaccine in two-year-olds - a nationwide cohort study Finland, influenza season 2015/16. Euro Surveill 2016;21:30346.

46. Ambrose CS. 2015-16 US Influenza Vaccine Effectiveness Influenza Clinical Investigation for Children (ICICLE) Study, 22 June 2016 https:// www.cdc.gov/vaccines/acip/meetings/downloads/slides-2016-06/influ enza-06-ambrose.pdf.

47. Flannery B, Chung J. Influenza Vaccine Effectiveness, Including LAIV vs. IIV in Children and Adolescents, US Flu VE Network, 2015-16, 22 June 2016 https://www.cdc.gov/vaccines/acip/meetings/downloads/slides2016-06/influenza-05-flannery.pdf.

48. Grohskopf LA, Sokolow LZ, Broder KR, et al. Prevention and control of seasonal influenza with vaccines. MMWR Recomm Rep 2016;65:1-54.

49. Grohskopf LA, Sokolow LZ, Broder KR, et al. Prevention and control of seasonal influenza with vaccines: recommendations of the Advisory Committee on Immunization Practices - United States, 2017-18 influenza season. MMWR Recomm Rep 2017;66:1-20.

50. Chung JR, Flannery B, Thompson MG, et al. Seasonal effectiveness of live attenuated and inactivated influenza vaccine. Pediatrics 2016;137: e20153279.

51. Flannery B, Clippard J, Zimmerman RK, et al. Early estimates of seasonal influenza vaccine effectiveness - United States, January 2015. MMWR Morb Mortal Wkly Rep 2015;64:10-5.

52. Skowronski DM, Chambers C, Sabaiduc S, et al. A perfect storm: impact of genomic variation and serial vaccination on low influenza vaccine effectiveness during the 2014-2015 season. Clin Infect Dis 2016;63:21-32.

53. Loeb M, Russell ML, Manning V, et al. Live attenuated versus inactivated influenza vaccine in Hutterite children: a cluster randomized blinded trial. Ann Intern Med 2016;165:617-24.

54. Caspard H, Mallory R, Yu J, et al. Live Attenuated Influenza Vaccine Effectiveness in Children from 2009 Through 2015-2016: A MetaAnalysis. Pediatric Academic Societies Annual Meeting. MedImmune, Gaithersburg, MD, USA; San Francisco, CA, 2017. 
55. Caspard H, Coelingh KL, Mallory RM, et al. Association of vaccine handling conditions with effectiveness of live attenuated influenza vaccine against H1N1pdm09 viruses in the United States. Vaccine 2016;34: 5066-72.

56. Epperson S, Blanton L, Kniss K, et al. Influenza activity - United States, 2013-14 season and composition of the 2014-15 influenza vaccines. MMWR Morb Mortal Wkly Rep 2014;63:483-90.

57. Siegrist C. Vaccine Immunology, 2017 (cited 11 May 2017) http://www. who.int/immunization/documents/Elsevier_Vaccine_immunology.pdf.

58. Ambrose CS, Bright H, Mallory R. Letter to the editor: potential causes of the decreased effectiveness of the influenza A(H1N1)pdm09 strain in live attenuated influenza vaccines. Euro Surveill 2016;21: 30394.

59. McLean HQ, Thompson MG, Sundaram ME, et al. Impact of repeated vaccination on vaccine effectiveness against influenza $\mathrm{A}(\mathrm{H} 3 \mathrm{~N} 2)$ and $\mathrm{B}$ during 8 seasons. Clin Infect Dis 2014;59:1375-85.

60. Ohmit SE, Petrie JG, Malosh RE, et al. Influenza vaccine effectiveness in households with children during the 2012-2013 season: assessments of prior vaccination and serologic susceptibility. J Infect Dis 2015;211: 1519-28.

61. Martinez-Baz I, Casado I, Navascues A, et al. Effect of repeated vaccination with the same vaccine component against 2009 pandemic influenza A(H1N1) virus. J Infect Dis 2017;215:847-55.

62. Advisory Committee on Immunization Practices (ACIP) Summary Report. Meeting Held 29-30 October 2014. Atlanta, GA: CDC, 2014 (cited 26 May 2017) https://www.cdc.gov/vaccines/acip/meetings/ downloads/min-archive/min-2014-10.pdf.

63. Flannery B. LAIV vs. IIV Effectiveness: Summary of Evidence Since 2009, Centers for Disease Control and Prevention Advisory Committee on Immunization practices (ACIP) Meeting 22-23 June 2016. Atlanta, GA: CDC https://www.cdc.gov/vaccines/acip/meetings/downloads/slides2016-06/influenza-07-flannery.pdf.

64. Caspard H, Belongia EA, Bernatoniene J, et al. Multicenter Study of the Effectiveness of Live Attenuated Influenza Vaccine and Inactivated Influenza Vaccine in Children in 2015-2016 in the United States and the United Kingdom, 2016. Poster presented at: Options for Influenza Meeting, 24-28 August 2016. Chicago, IL.

65. Cotter CR, Jin H, Chen Z. A single amino acid in the stalk region of the H1N1pdm influenza virus HA protein affects viral fusion, stability and infectivity. PLoS Pathog 2014;10:e1003831.

66. Penttinen PM, Friede MH. Decreased effectiveness of the influenza A (H1N1)pdm09 strain in live attenuated influenza vaccines: an observational bias or a technical challenge? Euro Surveill 2016;21:30350.

67. Bandell A, Woo J, Coelingh K. Protective efficacy of live-attenuated influenza vaccine (multivalent, Ann Arbor strain): a literature review addressing interference. Expert Rev Vaccines 2011;10:1131-41.

68. Victor JC, Lewis KD, Diallo A, et al. Efficacy of a Russian-backbone live attenuated influenza vaccine among children in Senegal: a randomised, double-blind, placebo-controlled trial. Lancet Glob Health 2016;4: e955-65.

69. Brooks WA, Zaman K, Lewis KD, et al. Efficacy of a Russian-backbone live attenuated influenza vaccine among young children in Bangladesh: a randomised, double-blind, placebo-controlled trial. Lancet Glob Health 2016;4:e946-54.
70. Block SL, Yi T, Sheldon E, et al. A randomized, double-blind noninferiority study of quadrivalent live attenuated influenza vaccine in adults. Vaccine 2011;29:9391-7.

71. Block SL, Falloon J, Hirschfield JA, et al. Immunogenicity and safety of a quadrivalent live attenuated influenza vaccine in children. Pediatr Infect Dis J 2012;31:745-51.

72. Sheldon EA, Jeanfreau R, Sliman JA, et al. Immunogenicity of a quadrivalent Ann Arbor strain live attenuated influenza vaccine delivered using a blow-fill-seal device in adults: a randomized, activecontrolled study*. Influenza Other Respir Viruses 2013;7:1142-50.

73. Ferris M. Clinical Review of Supplemental Biologics License Application for MedImmune's Quadrivalent Live Attenuated Influenza Vaccine (Q/ LAIV), 2012 (cited 29 May 2017) https://www.fda.gov/downloads/ biologicsbloodvaccines/vaccines/approvedproducts/ucm295380.pdf.

74. Bright H, Mallory. R Update on Status of Investigation of Reduced LAIV Effectiveness. Presentation at CDC Advisory Council on Immunization Practices (ACIP) Meeting, 22 February 2017 (cited 11 May 2017) https:// www.cdc.gov/vaccines/acip/meetings/downloads/slides-2017-02/influenza-04bright-mallory.pdf.

75. MedImmune, Internal Data, MedImmune. Speke UK 2017.

76. Salo H, Kilpi T, Sintonen H, et al. Cost-effectiveness of influenza vaccination of healthy children. Vaccine 2006;24:4934-1.

77. Damm O, Eichner M, Rose MA, et al. Public health impact and costeffectiveness of intranasal live attenuated influenza vaccination of children in Germany. Eur J Health Econ 2015;16:471-88.

78. Kwong JC, Pereira JA, Quach S, et al. Randomized evaluation of live attenuated vs. inactivated influenza vaccines in schools (RELATIVES) cluster randomized trial: Pilot results from a household surveillance study to assess direct and indirect protection from influenza vaccination. Vaccine 2015;33:4910-5.

79. Mohn KG, Bredholt G, Brokstad KA, et al. Longevity of B-cell and T-cell responses after live attenuated influenza vaccination in children. J Infect Dis 2015;211:1541-9.

80. Mohn KG, Brokstad KA, Pathirana RD, et al. Live attenuated influenza vaccine in children induces B-cell responses in tonsils. J Infect Dis 2016;214:722-31.

81. Mohn KGI, Zhou F, Brokstad KA, et al. Boosting of cross-reactive and protection-associated $\mathrm{T}$ cells in children after live attenuated influenza vaccination. J Infect Dis 2017;215:1527-35.

82. Ilyushina NA, Haynes BC, Hoen AG, et al. Live attenuated and inactivated influenza vaccines in children. J Infect Dis 2015;211:352-60.

83. Advisory Committee on Imunization Practices (ACIP) Summary Report. Meeting held 22-23 June 2016, pp 83-102. Atlanta, GA: CDC, 2016 (cited 30 May 2017) https://www.cdc.gov/vaccines/acip/meetings/downloads/ min-archive/min-2016-06.pdf.

84. ACIP Votes Down Use of LAIV for 2016-2017 Flu Season, 2016 (cited 31 May 2017) https://www.cdc.gov/media/releases/2016/s0622-laiv-flu.html.

85. FDA Information Regarding FluMist Quadrivalent Vaccine, 2016 (cited 1 June 2017). https://www.fda.gov/biologicsbloodvaccines/vaccines/ approvedproducts/ucm508761.htm.

86. Flu-Miffed: Piecing Together Clues on How FluMist Lost its Place in the Flu-Fighting Toolbox, 2016 (cited 25 May 2017) http://khn.org/news/flumiffed-piecing-together-clues-on-how-flumist-lost-its-place-in-the-flufighting-toolbox/. 